\title{
Ranging-free UHF-RFID Robot Positioning through Phase Measurements of Passive Tags
}

\author{
Valerio Magnago, Luigi Palopoli, Alice Buffi, Member, IEEE, Bernardo Tellini, Senior Member, IEEE, \\ Andrea Motroni, Student Member, IEEE, Paolo Nepa, Member, IEEE, David Macii, Senior Member, IEEE, and \\ Daniele Fontanelli, Senior Member, IEEE
}

\begin{abstract}
The phase of signals backscattered by Ultra High Frequency (UHF) Radio Frequency Identification (RFID) tags is generally more insensitive to multipath propagation than Received Signal Strength Indicator (RSSI). However, signal phase measurements are inherently ambiguous and could be further affected by unknown phase offsets added by the transponders. As a result, the localisation of an agent by using only signal phase measurements looks infeasible. In this paper, it is shown instead that the design of a dynamic position estimator (e.g. a Kalman filter) based only on signal phase measurement is actually possible. To this end, the necessary conditions to ensure theoretical local nonlinear observability are firstly demonstrated. However, a system that is locally observable guarantees convergence of the localisation algorithm only if the actual initial agent position is approximately known a priori. Therefore, the second part of the analysis covers the global observability, which ensures convergence starting from any initial condition in the state space. It is important to emphasise that complete observability holds only in theory. In fact, measurement uncertainty may greatly affect position estimation convergence. The validity of the analysis and the practicality of this localisation approach are further confirmed by numerical simulations based on an Unscented Kalman Filter (UKF).
\end{abstract}

Keywords-UHF-RFID, indoor localisation, RFID phase-based localisation, estimation algorithms, observability analysis.

\section{INTRODUCTION}

Indoor localisation is currently regarded as an enabling service for a variety of ICT applications. The set of possible indoor localisation techniques is really wide, as it includes solutions for both people and robot tracking in public environments or in industrial scenarios [1]-[3]. Boosted by these increasing application domains, several methods and sensing technologies were proposed for indoor localisation. In many applications, a given target positioning uncertainty has to be guaranteed with a high level of confidence [4], [5]. This result can be achieved by using anchor nodes or landmarks providing

Valerio Magnago and Luigi Palopoli are with the Department of Information Engineering and Computer Science, University of Trento, Via Sommarive 9, 38123, Trento, Italy, (e-mail: luigi.palopoli@unitn.it).

Andrea Motroni and Paolo Nepa are with the Department of Information Engineering, University of Pisa, Via Girolamo Caruso, 16, 56122, Pisa, Italy, (e-mail: andrea.motroni@ing.unipi.it).

Alice Buffi and Bernardo Tellini are with the Department of Energy, Systems, Territory and Constructions Engineering, Largo Lucio Lazzarino, 56122, Pisa, Italy, (e-mail: alice.buffi@unipi.it).

David Macii and Daniele Fontanelli are with the Department of Industrial Engineering, Via Sommarive 5, 38123, Trento, Italy (e-mail: alice.buffi@unipi.it). the moving agent with sporadic, but accurate information on position and/or orientation in a given reference frame. The most common technologies for indoor localisation rely on the Received Signal Strength Indicator of radio frequency (RF) (especially WiFi or Bluetooth) signals [6], [7], or on the Timeof-Flight (ToF) measurement of Ultra WideBand (UWB) [8][12], optical [13], [14] or ultrasonic signals [15]. An overview and a classification of the existing wireless indoor positioning solutions can be found in [16], [17]. Alternatively, in the case of robots, Laser Imaging Detection and Ranging (LiDAR) techniques can be effectively used [18].

Among the various radio frequency technologies proposed for indoor localisation, the use of RF IDentification (RFID) systems based on passive tags is particularly interesting due to their low cost and to their easy deployment [19]-[23]. When one or more tags, with known coordinates in a given reference frame, are detected by the RFID reader placed on the agent (e.g., a robot), the agent position can be estimated through dynamic estimation algorithms based on sensor fusion such as Kalman or particle filters. Beyond the tag detection data [24], the amplitude of the signals backscattered by multiple tags and received by te RFID reader (Received Signal Strength Indicator - RSSI) can be employed to estimate the agent position through multilateration or $k$-nearest neighbour algorithms, once the distance from the detected reference tags is measured [25]-[27]. Unfortunately, the distance data based on RSSI measurements are strongly affected by the multipath propagation and a reliable path loss model is difficult to derive, especially in scenarios affected by frequent changes. On the contrary, the phase of signals backscattered by tags is a more reliable parameter in rich multipath environments and, consequently, it is more suitable for localisation purposes [28], [29]. However, the inherent $2 \pi$ ambiguity of phase measurements as well as the unknown offset term due to the transponders and the reader and antenna components make the implementation of phase-based positioning algorithms rather challenging [30]. To address this problem, phase unwrapping techniques can be adopted if multiple readings with a proper spatial sampling are collected [31]. In [32], robot localisation is achieved through a multi-hypothesis Extended Kalman Filter (EKF) which combines the data from odometry sensors with the phase of the signals backscattered by the reference tags. The latter are custom tags placed on the ceiling. However, a calibration procedure for each reference tag is required to estimate the phase offset term. Such a method is extended in [33] by employing both RSSI and phase data. The new 
algorithm exhibits higher robustness when the knowledge of the reference tag positions is limited. Moreover, the phase offset calibration procedure can be avoided. Experimental results show that a robot can be localised with an error of about $4 \mathrm{~cm}$ in a $4 \mathrm{~m} \times 3 \mathrm{~m}$ room by employing just two reference tags. Finally, the authors state that the method can be employed in rooms of arbitrary size by deploying a reference tag every $2 \mathrm{~m}^{2}$. In [34] the authors propose a phase-based localisation method for mobile nodes equipped with a UHFRFID system with a low-density distribution of reference tags. The on-board reader collects phase data from a set of passive reference tags during the mobile node trajectory, by forming a synthetic array. Then, the phase data are combined with the information acquired by low-cost kinematic sensors through a sensor fusion approach. The method can be employed in both indoor and outdoor scenarios regardless of the reference tag positions.

An alternative possible solution is a nonlinear estimator reconstructing the state (namely the position of the agent) from the time series of the observations. However, the existence of a solution of this kind is not obvious. This paper addresses this problem as a whole, tackling both theoretical and practical aspects. The considered system is a unicycle robot equipped with RFID readers and odometric sensors. In order to localise itself the robot merges the measurement data from odometers (that provide information on its relative motion) with the readings of the RFID reader. However, instead of measuring the distance from the a set of RFID tags by using RSSI data, the proposed solution relies on the derivatives (or the finite differences in the discrete-time case) of such distances measured on the basis of the phase of the received signal regardless of the $2 \pi$ ambiguity typically affecting phase values. The main contributions of this paper are summarised below.

1) First, the local observability analysis proposed in [35] is recalled. The main purpose of this analysis is to show conditions for the existence of an estimator and for its convergence to the actual state when some a-priori information on the actual position is available (e.g., on the basis of probabilistic maps [36]). Since the analytical tools available are purely theoretical, continuous-time system and measurement models are adopted.

2) When no a-priori position information is available, a global observability analysis is needed. Due to the high complexity of this problem [37], [38], ad-hoc proofs based on geometric arguments for the global problem are provided. Moreover, we explicitly take into account the finite sampling time of a realistic sensing system.

3) Finally, we show and discuss the influence of measurement uncertainty on global observability, which is often disregarded in this class of problems. It turns out that when only the RFID tags are detected, it could be impossible to reconstruct the path if the robot follows a straight line because the measurements produced by two symmetric lines are exactly the same. For more general trajectories, we show that even if the path followed is theoretically observable, in practice state-of-the-art estimators such as the Unscented Kalman Filter could easily fail to converge (or could converge to a wrong estimated path) if the random measurement uncertainty contributions are so large as to make the trajectories indistinguishable [39]. In these cases, at least an additional tag is needed to ensure global convergence.

To the best of the Authors' knowledge, this is the first theoretical analysis presented on this problem, which is further complemented by a practical dynamic position tracking estimator for wheeled robots based on an Unscented Kalman Filter (UKF) to confirm the correctness of the proposed analysis. It is worth emphasizing that the use of phase-based measurements of the derivative of the distances (rather than the distance values themselves) between mobile agent and UHF-RFID tags, makes the proposed approach completely different and hardly comparable with other existing techniques.

The rest of the paper is organised as follows. Section II defines system and measurement models. Section III recalls the local observability analysis. Section IV is focused on global observability analysis once the original system is discretised and measurement uncertainty is considered. A position tracking estimator based on an Unscented Kalman Filter (UKF) for wheeled robots equipped with odometers and a single-antenna RFID reader is used to confirm the correctness of the proposed analysis. The corresponding simulation results are reported in Section V. Finally, in Section VI the main conclusions are drawn and the future work is outlined.

\section{PROBlem Formulation AND MOdELS}

The purpose of the method described in this paper is to track the position of a robot moving in an indoor environment where $n$ RFID tags are placed at known locations. The robot is assumed to be equipped with encoders on the rear wheels providing dead reckoning. Each sensor is supposed to be affected by uncertainties generated by white stochastic processes with known features. Even though the presented approach can be applied to a generic platform moving indoor on an horizontal plane using both ego-motion data and phase measurements of RF signals backscattered by passive RFID tags, the reported analysis refers to the case of the robotic walker FriWalk developed in the project ACANTO ${ }^{1}$.

\section{A. Platform model}

The FriWalk kinematic model is homologous to a unicyclelike robot. The pose of the wheeled vehicle at time $t$ is represented by triple $(x(t), y(t), \theta(t))$, where $(x(t), y(t))$ is the pair of planar coordinates of the mid-point of the rear axle expressed in the fixed right-handed reference frame $\langle W\rangle=\left\{O_{w}, X_{w}, Y_{w}, Z_{w}\right\}$. The FriWalk orientation at time $t$ is given by $\theta(t)$ that is the angle between the forwardoriented longitudinal symmetry axis of the robot and axis $X_{w}$. Thus, for localisation purposes, the robot state can be defined as $\mathbf{s}=[x, y, \theta]^{T} \in \mathcal{S}$, where the explicit reference to time variable $t$ is omitted for the sake of simplicity and $\mathcal{S}$ denotes the state space. The inputs to the system are $v$ and $\omega$, i.e. the

${ }^{1}$ EU Project ACANTO: A CyberphysicAl social NeTwOrk using robot friends - http://www.ict-acanto.eu/acanto, Feb. 2015 
forward and the angular velocities of the robot, respectively. The kinematic model is then given by

$$
\dot{\mathbf{s}}=G(\mathbf{s}) \mathbf{u}
$$

where

$$
G(\mathbf{s})=\left[\begin{array}{cc}
\cos (\theta) & 0 \\
\sin (\theta) & 0 \\
0 & 1
\end{array}\right]
$$

and $\mathbf{u}=[v, \omega]^{T}$. Observe that (1) is a driftless system.

\section{B. Measurement model}

As briefly explained at the beginning of Section II, FriWalk localisation relies on two measurement devices, i.e., a front UHF-RFID reader with a single antenna and two incremental encoders installed on the rear wheels. The angular velocities $\omega_{r}$ and $\omega_{l}$ of the right and left rear wheels can be expressed as a function of the robot forward and angular velocities $v$ and $\omega$, i.e.

$$
\omega_{r}=\frac{v}{r}+\frac{l \omega}{2 r} \text { and } \omega_{l}=\frac{v}{r}-\frac{l \omega}{2 r},
$$

where $r$ is the wheels radius and $l$ is the rear inter-axle length. If $\Delta_{r}=\omega_{r} T_{s}$ and $\Delta_{l}=\omega_{l} T_{s}$ denote the angular displacements of the right and left wheels, respectively, measured by the encoders in a sampling period $T_{s}$, by inverting (3), the incremental encoder measurement model for system (1) can be defined as

$$
\mathbf{h}^{e}\left(\Delta_{r}, \Delta_{l}\right)=\left[\begin{array}{l}
v T_{s} \\
\omega T_{s}
\end{array}\right]=\left[\begin{array}{l}
\frac{r}{2}\left(\Delta_{r}+\Delta_{l}\right) \\
\frac{r}{l}\left(\Delta_{r}-\Delta_{l}\right)
\end{array}\right] .
$$

The RFID reader is able to measure the phase of the signals backscattered by each UHF-RFID tag [21]. Therefore, if $\lambda$ is the wavelength of the RF signal and

$$
d_{i}=\sqrt{\left(x-x_{i}\right)^{2}+\left(y-y_{i}\right)^{2}},
$$

is the distance, at a given time, between the RFID reader antenna installed on the moving agent and the $i$-th RFID tag with Cartesian coordinates $\left(x_{i}, y_{i}\right)$ in $\langle W\rangle$ (see Fig. 1), the phase delay of the signal received by the reader after being backscattered by the tag is

$$
\phi_{i}=\frac{4 \pi d_{i}}{\lambda}+\delta_{i}^{\phi}=\phi_{i}^{\prime}+2 \pi N+\delta_{i}^{\phi}
$$

where $\phi_{i}^{\prime}=\bmod \left(\phi_{i}, 2 \pi\right), N$ is the integer number of halfwavelengths within distance $d_{i}$ and finally $\delta_{i}^{\phi}$ is a phase offset due mainly to cables, reader and antenna components and transponder backscattering [30]. Unfortunately, in practice the unwrapped absolute phase $\phi_{i}$ cannot be measured directly. Therefore, $N$ is unknownable and the phased-based measured distance $d_{i}^{m}$ is inherently ambiguous, i.e.

$$
d_{i}^{m}=\frac{\phi_{i}^{\prime}}{4 \pi} \lambda+\delta_{i}^{d}
$$

where the uncertainty term $\delta_{i}^{d}=N \frac{\lambda}{2}+\frac{\lambda}{4 \pi} \delta_{i}^{\phi}$ in general can be very large, but it is also approximately constant for distance variations smaller than half wavelength. Therefore, $\dot{\delta}_{i}^{d} \approx 0$ and $\dot{d}_{i}=\dot{d}_{i}^{m} \approx \frac{\lambda}{4 \pi} \dot{\phi}_{i}$, i.e. the variation in time of the relative

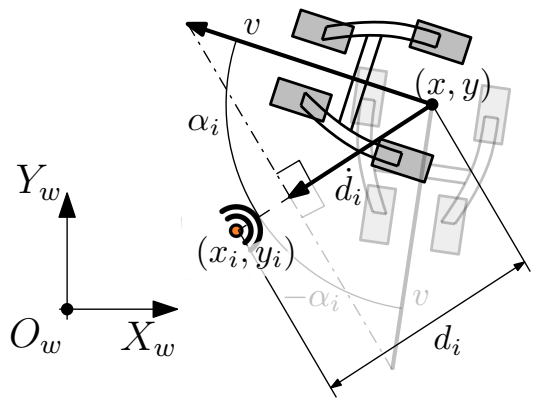

Fig. 1. Schematic representation of the FriWalk RFID measurement process assuming that the RFID antenna is placed in the middle of the rear wheels.

distance between the RFID reader and the $i-$ th tag tends to be insensitive to phase ambiguity.

In conclusion, by computing the derivative of (5) with respect to time, the measurement function adopted in the rest of this paper is

$$
h_{i}(\mathbf{s})=\dot{d}_{i}=\dot{d}_{i}^{m}=\frac{\cos (\theta)\left(x-x_{i}\right)+\sin (\theta)\left(y-y_{i}\right)}{\sqrt{\left(x-x_{i}\right)^{2}+\left(y-y_{i}\right)^{2}}} v .
$$

As depicted in Fig. 1, $\dot{d}_{i}^{m}$ is the component of the agent's forward velocity along the line connecting the point representing the FriWalk position (namely the mid-point of the rear wheels axle) and the point of the plane where the $i$-th tag is located. However, if $\alpha_{i}$ denotes the angle between $v$ and $d_{i}$ (as shown in Fig. 1) a single measurement based on (8) inherently returns ambiguous results because $\dot{d}_{i}=v \cos \left(\alpha_{i}\right)=v \cos \left(-\alpha_{i}\right)$.

\section{LOCAL OBSERVABILITY ANALYSIS}

The design of a robot position estimator for the problem described in Section II requires a preliminary local observability analysis to prove that the initial position of the robot $\mathbf{s}\left(t_{0}\right) \triangleq \mathbf{s}_{0}$ can be reconstructed by using both the sequence of input values (4) (namely the forward and angular velocity values returned by encoders) and the distance variations between robot and tags given by (8). In general, whenever the agent position cannot be reconstructed by using the measurement data available from a single fixed position (i.e. static observability), this analysis is mandatory. If the distance values are based on phase measurements only, multilateration cannot return a unique position even using an arbitrary number of RFID tags at known locations due to the unknown phase terms $\delta_{i}^{d}$ defined in Section II-B. Moreover, even if $\delta_{i}^{d}$ were known, the vehicle orientation $\theta$ could not be observed in any case. Thus, since system and measurement models are nonlinear, a proper nonlinear observability analysis is needed. In general, observability is a structural property of a system and it refers to the possibility to reconstruct the state of the system by observing its input-output behaviour. Therefore, if two different initial conditions exist for which the same input sequence generates the same output sequence, such initial conditions are indeed unobservable [38]. If the two initial conditions belong to the same neighbourhood of the 
state space, a local observability analysis can be performed; otherwise a global analysis is needed.

In formal terms, given vector $\mathbf{h}(\mathbf{s})=\left[\mathbf{h}_{1}(\mathbf{s}), \ldots, \mathbf{h}_{n}(\mathbf{s})\right]^{T}$ consisting of $n$ nonlinear measurement functions (8) associated with distinct RFID tags, the system is observable if $\mathbf{s}_{0}$ can be uniquely determined from $\mathbf{h}\left(\mathbf{s}_{0}\right)$ and its derivative at time $t_{0}$ [40]. The time derivatives of vector fields extracted from $G(\mathbf{s})$ in (2) are obtained through Lie-differentiation [38]. The local nonlinear observability just requires to have a locally injective function from the observation space (given by $\mathbf{h}(\mathbf{s})$ and its derivative) to the robot state space.

Since the problem with one tag only is clearly unobservable, the analytical analysis reported in [35] for two tags (assuming for instance that they are placed on the $Y_{w}$ axis at $\left(x_{1}, y_{1}\right)=$ $(0,-l)$ and $\left.\left(x_{2}, y_{2}\right)=(0, l)\right)$, showed that when the robot moves over a straight line, namely when $y=x \tan (\theta)+l$ or $y=x \tan (\theta)-l$ and $\omega=0$ in (1), a local injective function cannot be defined, i.e., the same measurement data (assuming negligible uncertainty) are potentially collected in both configurations due to their symmetry. While this problem persists for the global observability presented in Section IV, it is possible to show that local observability can be achieved by using geometric arguments [35]. Therefore, the trajectory followed by the agent is locally unobservable (i.e., an estimator cannot be designed even using strong a-priori information) only when the distance between the RFID tags is negligible compared to the distance from the agent or when the robot moves along the straight line joining the two passive RFID tags (indeed, in this case the same measurements based on (8) are collected). One additional outcome of this analysis (here not reported for space constraints), is that the second condition becomes observable when at least three non-collinear RFID tags are used. However, this is not the case for the first condition, as confirmed by the simulation results reported in Section V.

\section{Actual Global ObSERVABility For Two RFIDs}

The analysis reported above refers to the idealised situation in which the system and the observer evolution are timecontinuous. In a more realistic scenario, the evolution of the system and the measurements from the RFID tags can be observed at discrete-time instants. In this case, it is useful to write the system dynamics under the reasonable assumption that the angular velocity and the tangential velocity remain constant throughout a sampling period $T_{s}$, yielding the following discrete-time dynamics:

$$
\begin{aligned}
& x\left((k+1) T_{s}\right)=x\left(k T_{s}\right)+\int_{k T_{s}}^{(k+1) T_{s}} v \cos (\theta(\tau)) d \tau \\
& y\left((k+1) T_{s}\right)=y\left(k T_{s}\right)+\int_{k T_{s}}^{(k+1) T_{s}} v \sin (\theta(\tau)) d \tau \\
& \theta\left((k+1) T_{s}\right)=\theta\left(k T_{s}\right)+\omega T_{s} .
\end{aligned}
$$

Introducing for a generic function $f(\cdot)$ the notation $f_{k}=$ $f\left(k T_{s}\right)$, using the simple variable transformation $\tau^{\prime}=\tau-k T_{s}$ and considering basic trigonometric properties, it follows that the first equation in (9) becomes

$$
\begin{aligned}
& \int_{0}^{T_{s}} v \cos \left(\theta\left(\tau^{\prime}+k T_{s}\right)\right) d \tau^{\prime}= \\
& \begin{cases}v T_{s} \cos \theta_{k} & \text { if } \omega=0 \\
2 \frac{v}{\omega} \sin \left(\frac{\omega}{2} T_{s}\right) \cos \left(\theta_{k}+\frac{\omega}{2} T_{s}\right) & \text { otherwise. }\end{cases}
\end{aligned}
$$

Using the simplified notation $x_{k+1}=x\left((k+1) T_{s}\right)$ (i.e. $\mathbf{s}_{k+1}=$ $\left.\left[x_{k+1}, y_{k+1}, \theta_{k+1}\right]^{T}\right)$ and applying the same argument to the second integral of (9), it results that

$$
\begin{aligned}
x_{k+1} & = \begin{cases}x_{k}+v T_{s} \cos \theta_{k} \\
x_{k}+2 \frac{v}{\omega} \sin \left(\frac{\omega}{2} T_{s}\right) \cos \left(\theta_{k}+\frac{\omega}{2} T_{s}\right) & \text { otherwise }\end{cases} \\
y_{k+1} & = \begin{cases}y_{k}+v T_{s} \sin \theta_{k} & \text { if } \omega=0 \\
y_{k}+2 \frac{v}{\omega} \sin \left(\frac{\omega}{2} T_{s}\right) \sin \left(\theta_{k}+\frac{\omega}{2} T_{s}\right) & \text { otherwise }\end{cases} \\
\theta_{k+1} & =\theta_{k}+\omega T_{s} .
\end{aligned}
$$

Thus, the discrete-time version of measurement function (8) is the finite difference

$$
h_{i}\left(\mathbf{s}_{k}, \mathbf{s}_{k-1}\right)=d_{i}\left(\mathbf{s}_{k}\right)-d_{i}\left(\mathbf{s}_{k-1}\right),
$$

where $d_{i}\left(\mathbf{s}_{k}\right)=\sqrt{\left(x_{k}-x_{i}\right)^{2}+\left(y_{k}-y_{i}\right)^{2}}$ is the Euclidean distance between the agent and the $i-t h$ tag at the $k$-th sampling time.

In the rest of this section, we will extend the ideal local observability analysis to the more-realistic discretised global observability and provide technical evidence of our findings in two distinctive scenarios, chosen as a direct consequence of the discrete dynamics (10).

\section{A. The case of linear trajectories}

In Section III, we reported the two tags result stating that the trajectories are locally observable except for a few singular configurations. Unfortunately, additional singular configurations exist if we consider global observability. Of course, if the system is locally unobservable, it will be globally unobservable as well, while the converse is not necessarily true. A first negative result on global observability can be stated for linear trajectories.

Theorem 1: Consider system (10). The trajectories generated by the system for $\omega=0$ are not globally observable.

Proof: Consider the case in which two tags are deployed along the $Y_{w}$ axis, i.e. tags coordinates are $\left(0, y_{i}\right)$ for $i=1,2$. This is not a loss of generality because any system can be reduced to this condition through a simple transformation of coordinates. With reference to Fig. 2, suppose that the agent position is $U_{1}$ at step $k-1$ and moves to point $U_{2}$ at step $k$ (dash-dotted line). In this example, $d_{i}\left(\mathbf{s}_{k-1}\right)$, for $i=1,2$ is the length of segment $\overline{\operatorname{Tag}_{i} U_{1}}$, while $d_{i}\left(\mathbf{s}_{k}\right)$ is the length of segment $\overline{\operatorname{Tag}_{i} U_{2}}$. Therefore, the measurement functions $h_{i}\left(\mathbf{s}_{k}, \mathbf{s}_{k-1}\right)$ in (11) for $i=1,2$ return the length of the two segments $\overline{\operatorname{Tag}_{1} U_{2}}-\overline{\operatorname{Tag}_{1} U_{1}}=\overline{H_{1} U_{2}}$ and $\overline{\operatorname{Tag}_{2} U_{2}}-\overline{\operatorname{Tag}_{2} U_{1}}=\overline{H_{2} U_{2}}$. Observe that if the robot moves along an alias trajectory (dashed line), i.e. from point $V_{1}$ to point $V_{2}$, the same sequence of measurement data is 


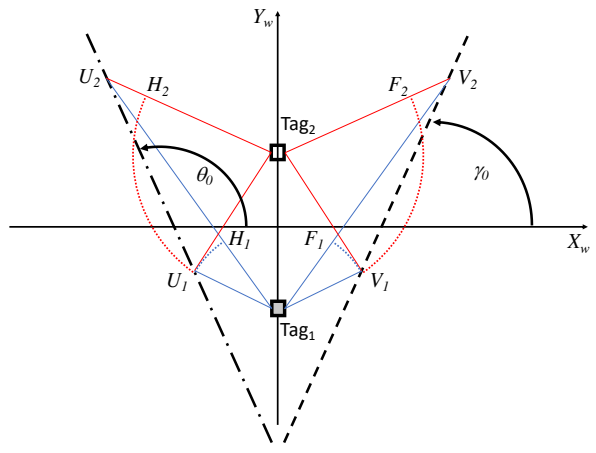

Fig. 2. Evolution of the system along a linear trajectory (dash-dotted line) and its alias trajectory (dashed line) producing the same measurement values for (11).

potentially collected, being the length of segments $\overline{F_{1} V_{2}}$ and $\overline{F_{2} V_{2}}$ respectively equal to $\overline{H_{1} U_{2}}$ and $\overline{H_{2} U_{2}}$. Of course, similar considerations hold when the robot moves straight from point $U_{2}$ (and the specular one $V_{2}$ ) onwards. In summary, if the robot moves along a line, the measurement data collected along a specular path with respect to the line joining the two RFID tags ideally would be the same. Therefore, the two linear trajectories are indistinguishable by using only the available measurement data.

Remark 1: The same result is easy to see using simple algebraic considerations. Indeed, given (10), the discrete time evolution along a line can be written as

$$
x_{k}=x_{0}+k v T_{s} \cos \theta_{0} \text { and } y_{k}=y_{0}+k v T_{s} \sin \theta_{0},
$$

with measurement functions

$h_{i}\left(\mathbf{s}_{k}, \mathbf{s}_{k-1}\right)=\sqrt{\left(x_{0}+k v T_{s} \cos \theta_{0}-x_{i}\right)^{2}+\left(y_{0}+k v T_{s} \sin \theta_{0}-y_{i}\right)^{2}}+$ $-\sqrt{\left(x_{0}+(k-1) v T_{s} \cos \theta_{0}-x_{i}\right)^{2}+\left(y_{0}+(k-1) v T_{s} \sin \theta_{0}-y_{i}\right)^{2}}$

for $i=1,2$. Being $x_{i}=0$ (tags on the $Y_{w}$ axis), we can see that the values of $h_{i}\left(\mathbf{s}_{k}, \mathbf{s}_{k-1}\right)$ remain point-wise equal $\forall k$ if we switch the initial state from $\left(x_{0}, y_{0}, \theta_{0}\right)$ to $\left(-x_{0}, y_{0}, \gamma_{0}\right)$, where $\cos \left(\theta_{0}\right)=-\cos \left(\gamma_{0}\right)$ (i.e., symmetric position w.r.t. the $Y_{w}$ axis, see Fig. 2).

To conclude, whenever the vehicle moves along a straight line taking measurements from two tags only, it is globally unobservable, i.e., any dynamic position estimator will inevitably fail in globally localising the agent in the plane of motion.

\section{B. The case of circular trajectories}

Following the same principle of Remark 1, we can verify if the same problem occurs for circular trajectories, i.e. $\omega \neq 0$ and constant in (10). Through some algebraic steps, the system evolution can be explicitly written as

$$
\begin{aligned}
& x_{k}=x_{0}+a \cos (\psi)-a \cos \left(k \omega T_{s}+\psi\right), \\
& y_{k}=y_{0}+a \sin (\psi)-a \sin \left(k \omega T_{s}+\psi\right),
\end{aligned}
$$

where $a=2 \frac{v}{\omega} \sin \left(\omega \frac{T_{s}}{2}\right) \frac{1}{\sqrt{2-2 \cos \left(\omega T_{s}\right)}}$ and $\psi=\omega \frac{T_{s}}{2}+\theta_{0}-$

\begin{tabular}{|c|c|c|c|c|}
\hline \multirow{4}{*}{ 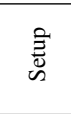 } & \multicolumn{4}{|c|}{ RFID 4} \\
\hline & \multicolumn{3}{|c|}{ RFID 3} & \\
\hline & \multicolumn{2}{|c|}{ RFID 2} & & \\
\hline & RFID 1 & & & \\
\hline & Tag 1 & Tag 2 & Tag 3 & Tag 4 \\
\hline$x[\mathrm{~m}]$ & 0 & 0 & 5 & -5 \\
\hline$y[\mathrm{~m}]$ & 5 & -5 & 0 & 0 \\
\hline
\end{tabular}
$\operatorname{atan}\left(-\sin \left(\omega T_{s}\right), 1-\cos \left(\omega T_{s}\right)\right)$.
TABLE I. SETUP SCENARIOS AND RFID TAG POSITIONS.

Letting $\bar{x}_{0}=x_{0}+a \cos (\psi)$ and $\bar{y}_{0}=y_{0}+a \sin (\psi)$ in (14), the expression for the measurement function $h_{i}\left(\mathbf{s}_{k}, \mathbf{s}_{k-1}\right)$ in (11) for a generic tag in $\left(0, y_{i}\right)$ (recall that without loss of generality the tags are placed along the $Y_{w}$ axis) is given by

$$
\begin{aligned}
& h_{i}\left(\mathbf{s}_{k}, \mathbf{s}_{k-1}\right)= \\
& \sqrt{\left[\bar{x}_{0}-a \cos \left(k \omega T_{s}+\psi\right)\right]^{2}+\left[\bar{y}_{0}-a \sin \left(k \omega T_{s}+\psi\right)-y_{i}\right]^{2}}- \\
& \sqrt{\left[\bar{x}_{0}-a \cos \left((k-1) \omega T_{s}+\psi\right)\right]^{2}+\left[\bar{y}_{0}-a \sin \left((k-1) \omega T_{s}+\psi\right)-y_{i}\right]^{2}}
\end{aligned}
$$

Contrary to the case of linear trajectories, if we switch the initial state from $\mathbf{s}_{0}=\left[x_{0}, y_{0}, \theta_{0}\right]^{T}$ to the specular $\tilde{\mathbf{s}}_{0}=\left[-x_{0}, y_{0}, \gamma_{0}\right]^{T}$ (marked as $U_{1}$ and $V_{1}$ in Fig. 2), we do not obtain the same measurement for $h_{i}\left(\mathbf{s}_{k}, \mathbf{s}_{k-1}\right)$. Interestingly, because $\psi$ is an even function of $\omega$, the same measurement from $\mathbf{s}_{0}$ can only be obtained starting from $\tilde{\mathbf{s}}_{0}$ if $\omega$ is changed into $-\omega$. In other words, in order to obtain the same sequence of measurement data for two different and symmetric circular trajectories, the direction of rotation should be inverted. However, since we are dealing with a self-localisation problem, the sign of $\omega$ is assumed to be known (e.g., from encoder measurements). Thus, the global observability of circular trajectories is not compromised.

\section{Simulation Results}

To verify the validity of the previous analysis, in this Section the results obtained with a Bayesian position tracking algorithm are reported. In particular, due to the strong nonlinearity of both system and measurement models, a UKF (whose description is here omitted for space limits) is preferable [39]. In all the simulations, Gaussian zero-mean uncertainty contributions are considered.

\section{A. Local observability proof}

In a first set of simulations, the sensing range of the RFID reader is supposed to be unlimited and four different setups are considered, as subsumed in Tab. I. We use the notation "RFID $i$ " to denote the $i$-th configuration shown in Tab. I, including 1 , 2,3 or 4 tags respectively. The measurements based on (8) are sampled with a sampling period of $T_{s}=10 \mathrm{~ms}$. The standard deviations of forward and angular velocity fluctuations are $5 \mathrm{~cm} / \mathrm{s}$ and $0.17 \mathrm{rad} / \mathrm{s}$, respectively. Measurement standard uncertainty is set to $5 \mathrm{~cm} / \mathrm{s}$. In all tests, robot forward velocity is set to $v=1 \mathrm{~m} / \mathrm{s}$, while the state of the system is initialised randomly (using independent Gaussian probability density functions - PDFs) around a given value $\mathbf{s}_{0}$ with standard deviations equal to $0.5 \mathrm{~m}$ along axes $X_{w}$ and $Y_{w}$ and $0.17 \mathrm{rad}$ 


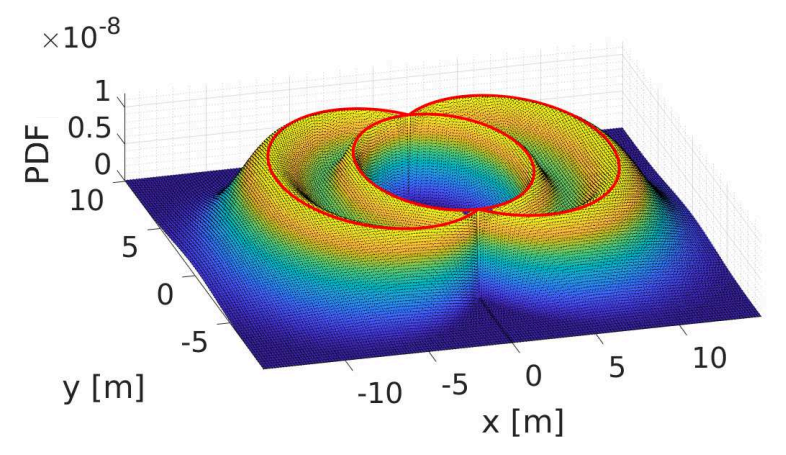

Fig. 3. Joint PDF for the $(x, y)$ Cartesian coordinates obtained from a single pair of measures with respect to two RFID tags.

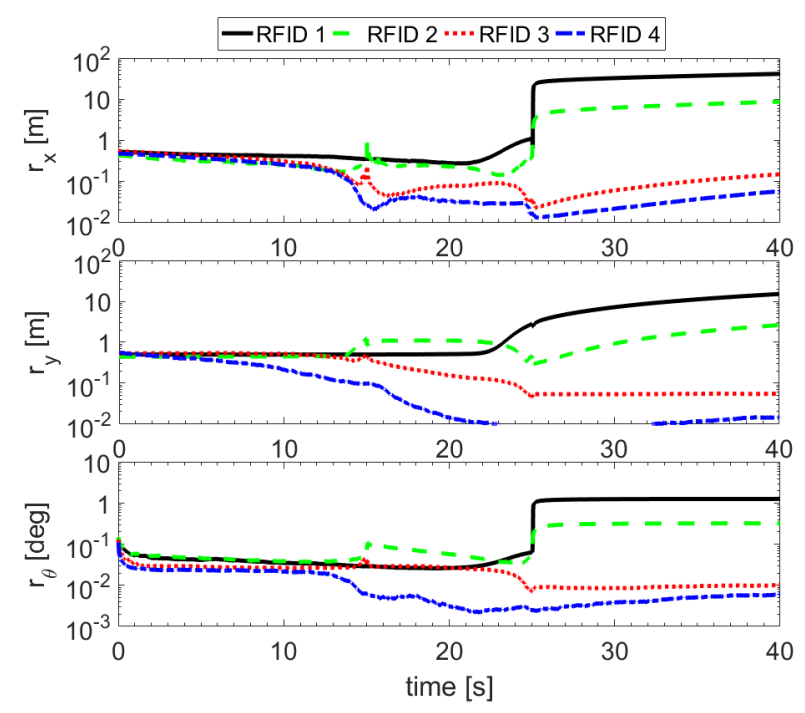

Fig. 4. RMSE values of the state variables estimated by the UKF in the four cases shown in Tab. I, when the robot moves straight along axis $Y_{w}$ (which is unobservable in the scenario with two tags).

as far as the orientation angle $\theta$ is concerned. To confirm the validity of the observability analysis reported in Section III, the joint posterior PDF was computed for a single pair of measures (i.e., $\mathbf{h}(\mathbf{s})=[-0.3162,-0.9487] \mathrm{m} / \mathrm{s}$ ) related to two RFID tags. By marginalising the joint PDF along $\theta$, the resulting bivariate PDF for the $(x, y)$ Cartesian coordinates is shown in Fig. 3.

In the first type of simulated trajectories, the agent moves straight (i.e., $\omega=0 \mathrm{rad} / \mathrm{s}$ ) along axis $Y_{w}$ from $[0,20,-\pi / 2]^{T}$ to $[0,-20,-\pi / 2]^{T}$ in $40 \mathrm{~s}$. The Root Mean Square Errors (RMSE) $r_{x}, r_{y}$ and $r_{\theta}$ associated with 100 estimates of state variables $x, y$ and $\theta$ are plotted in Fig. 4. Observe that at least three RFID tags are needed for UKF to converge (i.e., to reach small RMSE values), in accordance with the analysis reported in Section III.

In the second case study, the agent moves again along a straight line for $100 \mathrm{~s}$ (i.e., axis $X_{w}$ in this case) starting from $\mathbf{s}\left(t_{0}\right)=[-50,2,0]^{T}$ to $\mathbf{s}\left(t_{f}\right)=[50,2,0]^{T}$. The RMSE

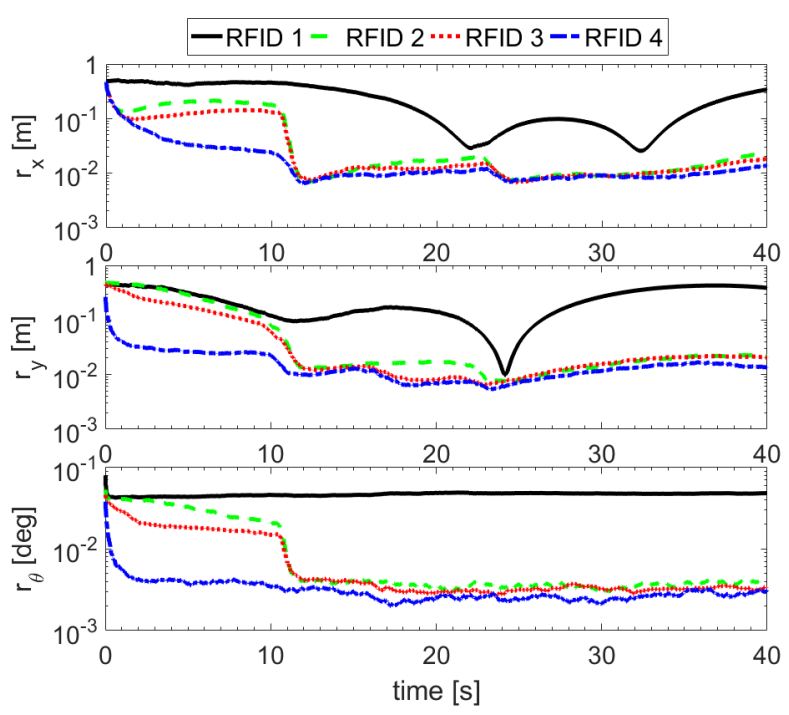

Fig. 5. RMSE values of the state variables estimated by the UKF in the four cases shown in Tab. I, when the robot moves straight along axis $X_{w}$.

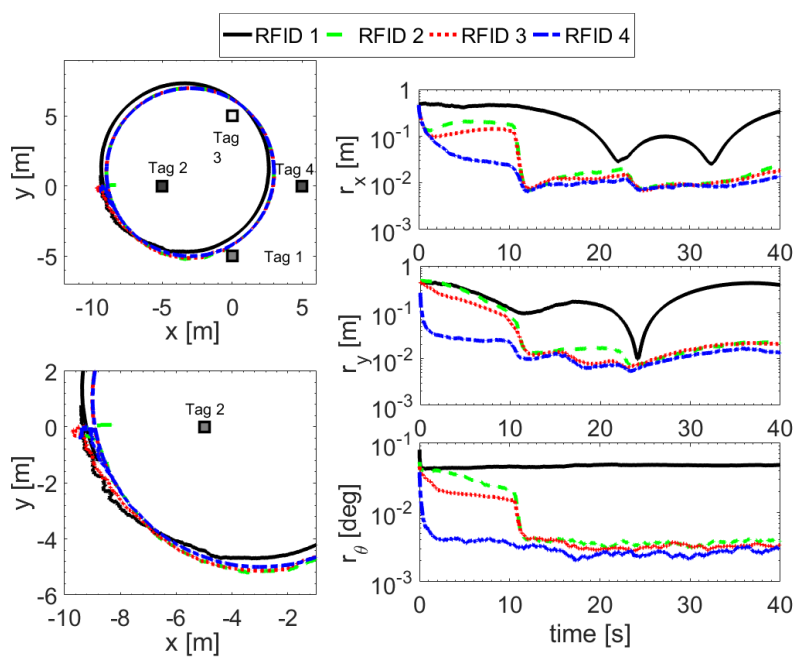

Fig. 6. On the left: Circular trajectory centred in $(-3,1) \mathrm{m}$ and with a radius of $6 \mathrm{~m}$ and excerpt of the estimated trajectory at the beginning of the motion. On the right: RMSE of the state variables estimated by the UKF in the four cases of Tab. I when the robot moves along the depicted circular trajectory.

values computed over 100 runs show that if one tag only is considered, the UKF does not converge, as expected (see Fig. 5). On the contrary, when at least two tags are used, the RMSE values tend asymptotically to small values (i.e., the UKF converges), thus confirming that the system is observable. Moreover, if $\omega \neq 0$, observability is always achieved even with two RFID tags. This is confirmed by the simulation results shown in Fig. 6 for a circular trajectory. Again, the RMSE values tend to diverge when just one RFID tag is considered (due to dead reckoning), whereas they tend to converge when 


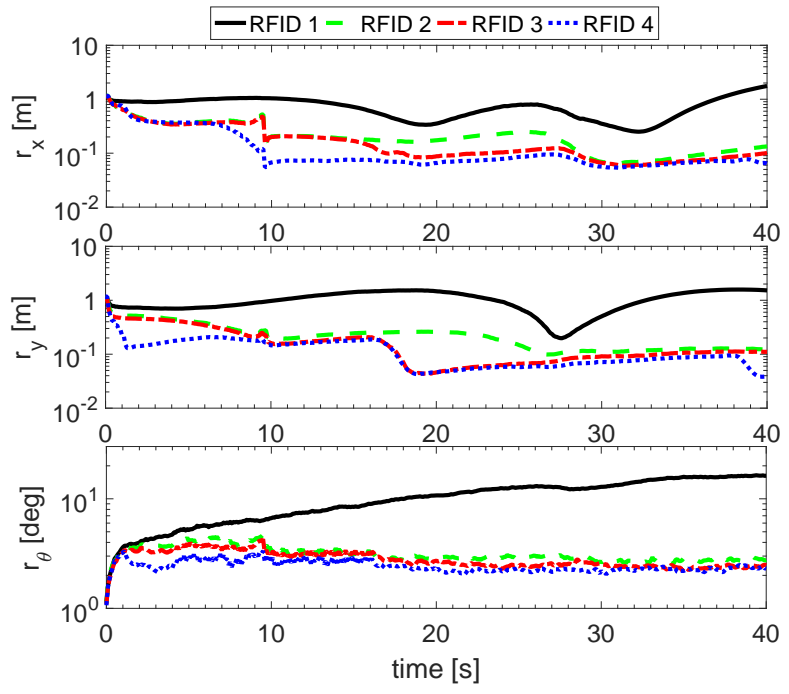

Fig. 7. RMSE of the state variables estimated by the UKF in the in the four tag setups shown in Tab. I when the robot moves along the circular trajectory of Fig. 6 .

at least two RFID tags are deployed.

\section{B. Global observability proof}

Following the same rationale of the proof for local observability, here the theoretical results of Section IV for global observability are verified through simulations. First the RMSE values for 100 trajectories in the same configurations presented in the previous Section are reported, although for different initial conditions of the UKF. Such conditions are randomly generated (using independent Gaussian PDFs) around a given value $\mathbf{s}_{0}$ with standard deviations equal to $1 \mathrm{~m}$ along axes $X_{w}$ and $Y_{w}$ and $\pi$ rad as far as the orientation angle $\theta$ is concerned. The standard deviations $\sigma_{v}=10 \mathrm{~cm} / \mathrm{s}$ for the longitudinal velocity $v$ and $\sigma_{\omega}=0.17 \mathrm{rad} / \mathrm{s}$ for the angular velocity $\omega$ are adopted, while the standard uncertainty of the phase-based measurement of the differences of two consecutive distance values between the RFID reader and any tag (in accordance with (11)) is set to $\sigma_{h}=10 \mathrm{~mm}$. Fig. 6 shows the estimated circular trajectories for the same RFID tag configurations reported in Tab. I, while Fig. 7 depicts the corresponding RMSE of the state variables estimated by the UKF. For linear trajectories moving along the axis, results similar to those visible in Fig. 4 and Fig. 5 have been obtained. Hence, they have been omitted for the sake of brevity. In general, the observability problem can be solved with more than two RFID tags, even though a slower convergence can be observed, especially as far as variable $\theta$ is concerned.

Nevertheless, as discussed in Section IV, the global observability analysis has indeed peculiar singular cases when two tags are adopted and needs a specific study. In the example shown in Fig. 8, the UKF is applied to a unicycle robot moving along a linear trajectory obtained by setting
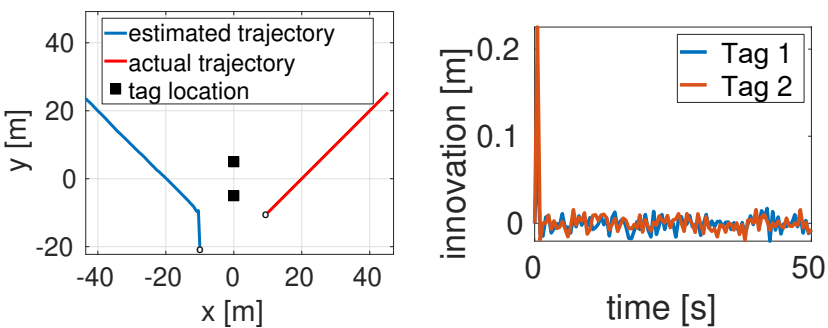

(A)
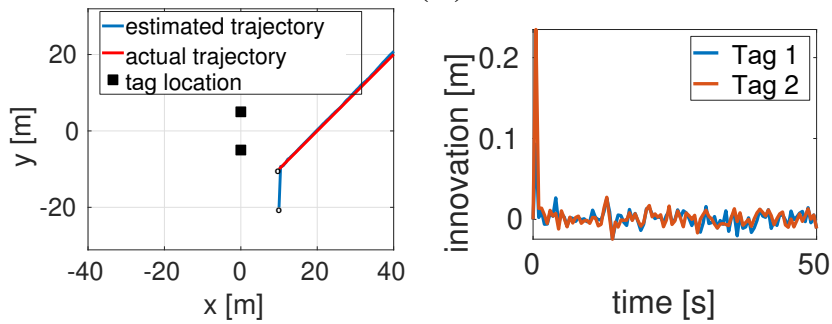

(B)

Fig. 8. Agent straight-line trajectory with initial conditions $\left(x_{0}, y_{0}\right)=$ $(10,-10) \mathrm{m}$ and $\theta_{0}=\pi / 4 \mathrm{rad}$. In the scenario A (top graphs) the UKF is initialised with $(-10,-20,3 \pi / 4)$, while in the scenario B (bottom graphs) the UKF is initialised with $(10,-20, \pi / 4)$. In both cases the starting points of the actual and estimated trajectories are highlighted with small circle markers. Also, the innovation terms (graphs on the right side) tend towards zero even if the estimates are different and may wrongly refer to the trajectory symmetric to the actual one.

$\omega=0 \mathrm{rad} / \mathrm{s}$ in (10). In this example, the forward velocity of the robot is $v=1 \mathrm{~m} / \mathrm{s}$. We assume that the inputs of the discrete system (10) are affected by zero mean, white Gaussian process noises with standard deviation of $\sigma_{v}=1 \mathrm{~cm} / \mathrm{s}$ for the longitudinal velocity $v$ and $\sigma_{\omega}=0.035 \mathrm{rad} / \mathrm{s}$ for the angular velocity $\omega$. The measurement uncertainty contributions affecting (11) exhibit also a Gaussian PDF with standard deviation $\sigma_{h}=5 \mathrm{~mm}$. The initial state $\mathbf{s}_{0}$ of the system (i.e., $(10 \mathrm{~m},-10 \mathrm{~m}, \pi / 4 \mathrm{rad}))$ is highlighted by circular markers. The sampling time is $T_{s}=50 \mathrm{~ms}$. In all the simulations reported for this Section, the RFID tags labeled as 1 and 2 in Tab. I are considered.

The UKF was initialised with two different set of states, which are also highlighted by circular markers. As clearly shown in Fig. 8, in scenario A, whose UKF initial state is $(-10 \mathrm{~m},-20 \mathrm{~m}, 3 \pi / 4 \mathrm{rad})$, the estimated state converges to the line symmetric to the actual trajectory with respect to the line connecting the two tags. In scenario $\mathrm{B}$, with initial state $(10 \mathrm{~m},-20 \mathrm{~m}, \pi / 4 \mathrm{rad})$, the estimated trajectory converges to the actual one. In both cases the innovation terms (namely, the differences between the measurement data and the corresponding values based on the predicted states) converge to zero for both tags. Therefore, even if both trajectories are perfectly consistent with the model, but the position of the agent (i.e. the state of the system) is clearly globally unobservable.

On the other hand, even if the circular paths are theoretically globally observable, measurement uncertainty may completely disrupt estimation results. Consider, for instance, the scenario in which an agent moves along the circular 


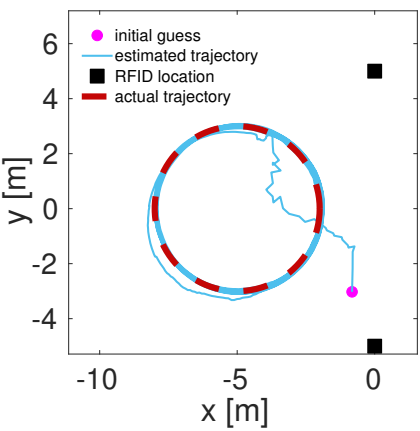

(A)

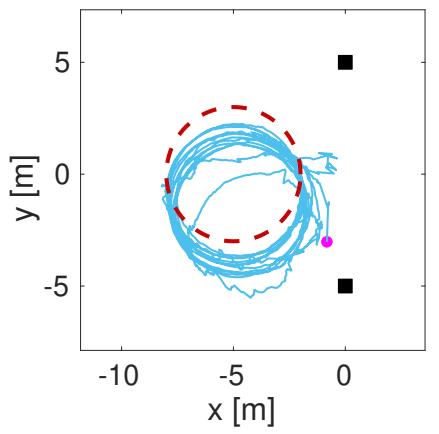

(B)
Fig. 9. Agent circular path and corresponding trajectory estimated by the UKF for a small measurement standard uncertainty (scenario A with $\sigma_{h}=$ $5 \mathrm{~mm}$ ) and a higher measurement standard uncertainty (scenario B with $\sigma_{h}=$ $100 \mathrm{~mm}$ ). In practice, measurement uncertainty may prevent convergence.
(A)

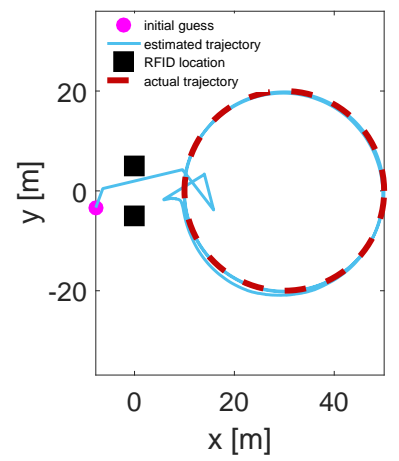

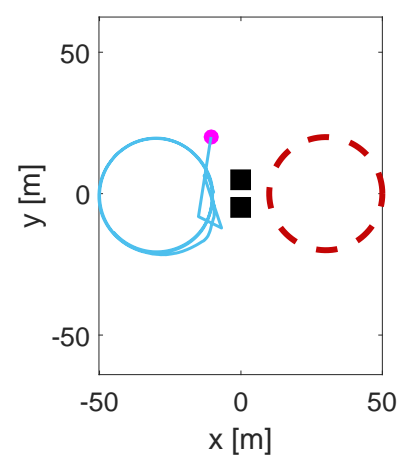

(B)
Fig. 10. Agent circular path and corresponding trajectory estimated by the UKF when the radius is large (i.e., about $20 \mathrm{~m}$ ). In scenario A (on the left), the UKF is initialised with a state close to the actual trajectory. In scenario B (on the right) the UKF is initialised with a state close to the specular trajectory.

trajectory depicted with a dashed line in Fig. 9. In this case, the forward and angular velocities of the agent are: $v=1 \mathrm{~cm} / \mathrm{s}$ and $\omega=0.0175 \mathrm{rad} / \mathrm{s}$, respectively. Measurement standard uncertainty changes from scenario A (where it is very low, i.e. $\sigma_{h}=5 \mathrm{~mm}$, like in the previous case), to scenario B, where it reaches $\sigma_{h}=100 \mathrm{~mm}$. The initial state of the system is $(-2 \mathrm{~m}, 0 \mathrm{~m}, \pi / 2 \mathrm{rad})$, while in both scenarios the UKF is initialised with $(-0.8 \mathrm{~m},-3 \mathrm{~m}, 1.6 \mathrm{rad})$.

In Scenario A, the estimated trajectory quickly converges to the ideal one (i.e., perfectly circular), as shown in the left part of Fig. 9. This behaviour was consistently observed in multiple simulation trials. In Scenario B, the estimates generated by the UKF converge instead to a circle which is shifted down by roughly $1.5 \mathrm{~m}$ (as shown in the right part of the Fig. 9). As discussed in Section IV, the existence of different equilibria of the UKF confirms that the practical global observability depends on the specific measurement functions adopted.

The case shown in Fig. 10 for $v=1 \mathrm{~m} / \mathrm{s}$ and $\omega=0.05 \mathrm{rad} / \mathrm{s}$ is even more interesting. These parameters produce an ideal circular trajectory with radius equal to $20 \mathrm{~m}$ (still depicted with a dashed line). In this example, standard uncertainty values are: $\sigma_{\omega}=0.02 \mathrm{rad} / \mathrm{s}, \sigma_{v}=10 \mathrm{~cm} / \mathrm{s}$ and $\sigma_{h}=0.1 \mathrm{~mm}$. In this case, the standard deviation of the process noise related to the odometry measurements is quite high compared to $\sigma_{h}$. In both tests reported in the figure, the actual robot initial state was $(10 \mathrm{~m}, 0 \mathrm{~m},-\pi / 2 \mathrm{rad})$. However, the UKF was initialised with a different state. In Scenario A (left of the figure) the filter was initialised with $(-7.75 \mathrm{~m},-3.35 \mathrm{~m},-1.72 \mathrm{rad})$. In Scenario B (right of the figure) the filter was initialised with $(-10.59 \mathrm{~m},-20.03 \mathrm{~m},-1.85 \mathrm{rad})$. The behaviour in the two cases is quite different: in Scenario A the filter tracks quite precisely the state, while in Scenario B the estimated state settles down on the circle that is symmetric with respect to the line joining the two RFID tags. This is surprising because, as explained in Section (IV-B), the same measurement data can return two symmetric circular trajectories only if the agent moves in opposite directions (clockwise vs. counterclockwise), which instead does not happen in the case considered. The explanation of this anomalous behaviour is based on the Bayesian nature of the UKF: the high level of uncertainty on $\omega$ leads the UKF to rely mainly on the distance variation measurements instead of the odometry data. As a consequence, the updates generated by $\omega$ are virtually cancelled by the correction generated by the RFID-based measurement data. As a result, it may happen that the estimated trajectory refers to a circular motion in the opposite direction with respect to the actual one. A possible (unintuitive) counteraction to avoid this problem is to increase $\sigma_{h}$ so that the odometry becomes "more credible", thus shifting the estimates toward the actual path.

\section{Realistic scenario}

To complete the analysis, the results of some simulations in a more complex and realistic scenario based on the features of the Friwalk platform are reported in this Section. The rear wheels of the Friwalk are equipped with two incremental encoders AMT-102V with a resolution of up to 2048 pulse per revolution (PPR). In practice, these data are collected by a BeagleBone black board via a Controller Area Network (CAN) bus. From a preliminary characterization of both encoders, we found that, when the robot moves at speed in the order of $1 \mathrm{~m} / \mathrm{s}$, the standard uncertainty associated with angular and forward velocities are $\sigma_{\omega} \approx 0.01 \mathrm{rad} / \mathrm{s}$ and $\sigma_{v} \approx 5 \mathrm{~cm} / \mathrm{s}$, respectively.

An onboard Impinj Speedway Revolution R420 UHF-RFID reader equipped with a CAEN RFID WANTENNA X019 is able to detect UHF signals $(865-868 \mathrm{MHz})$ backscattered by passive tags within a range of around $5 \mathrm{~m}$ from the reader (e.g., with a receiver sensitivity approximately equal to $-18 \mathrm{dBm}$ ). Based on the specifications of the UHF-RFID kit, the standard uncertainty associated with the difference of subsequent distance measurement is $\sigma_{h}=5.5 \mathrm{~mm}$.

The RFID tags are supposed to be placed over squarepatterned regular grids of different density. In particular, the distance between pairs of adjacent tags ranges from $1 \mathrm{~m}$ to $5 \mathrm{~m}$. A total of 100 random robot trajectories was generated in a $100 \mathrm{~m} \times 10 \mathrm{~m}$ corridor by using a path planning algorithm conceived for assistive robots [41]. In every run, the robot starts from a randomly chosen point on one side of the corridor and reaches the other side in about $100 \mathrm{~s}$ moving mainly (but 

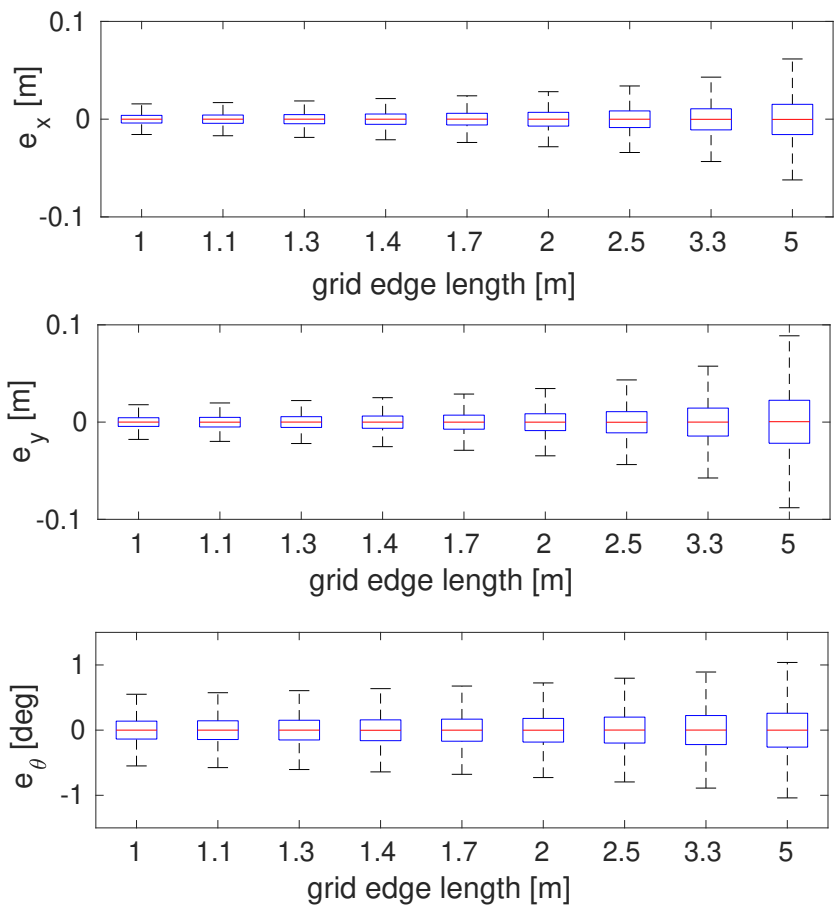

Fig. 11. Box-and-whiskers plots of the localisation errors computed over 100 trajectories of a robot moving randomly in a $100 \mathrm{~m} \times 10 \mathrm{~m}$ corridor for different distances between pairs of adjacent RFID tags placed over squarepatterned regular grids.

not perfectly) straight, while crossing different intermediate points. The box-and-whiskers plots of the aggregated UKF state estimation errors are shown in Fig. 11 for various edge lengths of the grid of RFID tags. The edges of each box refer to the 25th and 75th percentiles of the corresponding estimation errors, whereas the whiskers stretch to the 99th percentile.

In all cases, the random motion of the robot as well as the fact that more than two RFID tags are always detected, makes the Friwalk position steadily observable, as confirmed by the fact that the estimation errors are bounded and very small, i.e., between about $2.5 \mathrm{~cm}$ and $7 \mathrm{~cm}$ with $99 \%$ probability along axes $x$ and $y$ and generally below 1 degree (i.e. $0.0175 \mathrm{rad}$ ) in the worst-case as far as robot orientation $\theta$ is concerned. These results subsume the observability analysis and prove the practicality of the proposed localisation approach in realistic scenarios. Of course, localisation uncertainty tends to grow as the granularity of the grid of RFID tags become coarser. In fact, in this case, a lower number of measurements is available at every time step.

\section{CONCLUSIONS}

The distinctive challenging feature of localisation based on the phase of the RF signals backscattered by UHF-RFID tags is the inherent ambiguity of phase measurements themselves. We have presented a complete nonlinear observability analysis for this challenging problem. As a result of this analysis, singular configurations are identified and discussed. In particular, the system is locally observable using three non-collinear RFID tags, while for two tags, a small subset of straight-line paths is unobservable. For global observability, we have considered the critical case when just two tags are used. A first negative result is that the straight-line paths are always unobservable, no matter the amount of noise that affect each measurement. On the contrary, circular trajectories are globally observable (at least from the theoretical point of view). However, our empirical analysis shows the crucial role of measurement uncertainty. Specifically, a large uncertainty affecting RFID-based phase measurements hamper position estimator convergence, or even worse, could drive the estimated state towards a wrong path.

The validity of the analysis and the effectiveness of the localisation approach were verified using an ad-hoc Unscented Kalman Filter (UKF). The implementation and the field experimental validation of the position estimation algorithm, currently confirmed by some preliminary simulations in a real scenario, are the objectives of a future work.

\section{REFERENCES}

[1] A. Colombo, D. Fontanelli, D. Macii, and L. Palopoli, "Flexible Indoor Localization and Tracking based on a Wearable Platform and Sensor Data Fusion," IEEE Trans. on Instrumentation and Measurement, vol. 63, no. 4, pp. 864-876, Apr. 2014

[2] V. Kulyukin, A. Kutiyanawala, E. LoPresti, J. Matthews, and R. Simpson, "iWalker: Toward a rollator-mounted wayfinding system for the elderly," in Proc. IEEE Int. Conference on RFID, Las Vegas, NV, USA, Apr. 2008, pp. 303-311.

[3] P. Nazemzadeh, F. Moro, D. Fontanelli, D. Macii, and L. Palopoli, "Indoor Positioning of a Robotic Walking Assistant for Large Public Environments," IEEE Trans. on Instrumentation and Measurement, vol. 64, no. 11, pp. 2965-2976, Nov. 2015.

[4] V. Magnago, P. Bevilacqua, L. Palopoli, R. Passerone, D. Fontanelli, and D. Macii, "Optimal Landmark Placement for Indoor Positioning using Context Information and Multi-sensor Data," in Proc. IEEE Int. Instrumentation and Measurement Technology Conference (I2MTC), Houston, Texas (US), May 2018, pp. 1-6.

[5] V. Magnago, L. Palopoli, R. Passerone, D. Fontanelli, and D. Macii, "A Nearly Optimal Landmark Deployment for Indoor Localisation with Limited Sensing," in International Conference on Indoor Positioning and Indoor Navigation (IPIN), Sapporo, Japan, Sep. 2017, pp. 1-8.

[6] P. Chen, Y. B. Xu, L. Chen, and Z. A. Deng, "Survey of WLAN fingerprinting positioning system," Applied Mechanics and Materials, vol. 380, pp. 2499-2505, Aug. 2013.

[7] D. Giovanelli, E. Farella, D. Fontanelli, and D. Macii, "Bluetoothbased Indoor Positioning through ToF and RSSI Data Fusion," in Proc. International Conference on Indoor Positioning and Indoor Navigation (IPIN), Nantes, France, Sep. 2018, pp. 1-8.

[8] Y. Zhou, C. L. Law, Y. L. Guan, and F. Chin, "Indoor Elliptical Localization Based on Asynchronous UWB Range Measurement," IEEE Transactions on Instrumentation and Measurement, vol. 60, no. 1, pp. 248-257, Jan. 2011.

[9] A. Cazzorla, G. De Angelis, A. Moschitta, M. Dionigi, F. Alimenti, and P. Carbone, "A 5.6-GHz UWB Position Measurement System," IEEE Transactions on Instrumentation and Measurement, vol. 62, no. 3, pp. 675-683, Mar. 2013.

[10] A. R. Jiménez Ruiz and F. Seco Granja, "Comparing Ubisense, BeSpoon, and DecaWave UWB Location Systems: Indoor Performance Analysis," IEEE Transactions on Instrumentation and Measurement, vol. 66, no. 8, pp. 2106-2117, Aug. 2017.

[11] V. Magnago, P. Corbalán, G. Picco, L. Palopoli, and D. Fontanelli, "Robot Localization via Odometry-assisted Ultra-wideband Ranging with Stochastic Guarantees," in Proc. IEEE/RSJ International Conference on Intelligent Robots and System (IROS), Macao, China, Oct. 2019. 
[12] F. Lazzari, A. Buffi, P. Nepa, and S. Lazzari, "Numerical Investigation of an UWB Localization Technique for Unmanned Aerial Vehicles in Outdoor Scenarios," IEEE Sensors Journal, vol. 17, no. 9, pp. 28962903, May 2017.

[13] R. Mautz and S. Tilch, "Survey of optical indoor positioning systems," in Proc. Int. Conference on Indoor Positioning and Indoor Navigation (IPIN), Guimarães, Portugal, Sep. 2011, pp. 1-7.

[14] P. Nazemzadeh, D. Fontanelli, D. Macii, and L. Palopoli, "Indoor Localization of Mobile Robots through QR Code Detection and Dead Reckoning Data Fusion," IEEE/ASME Transactions on Mechatronics, vol. 22, no. 6, pp. 2588-2599, Dec. 2017.

[15] F. Ijaz, H. K. Yang, A. Ahmad, and C. Lee, "Indoor positioning: A review of indoor ultrasonic positioning systems," in 15th Int. Conference on Advanced Communication Technology (ICACT), Phoenix Park, PyeongChang, South Korea, Jan. 2013, pp. 1146-1150.

[16] H. Liu, H. Darabi, P. Banerjee, and J. Liu, "Survey of wireless indoor positioning techniques and systems," IEEE Transactions on Systems, Man, and Cybernetics, Part C (Applications and Reviews), vol. 37, no. 6, pp. 1067-1080, Nov. 2007.

[17] C. Li, L. Mo, and D. Zhang, "Review on UHF RFID Localization methods," IEEE Journal of Radio Frequency Identification, vol. 3, no. 4, pp. 205-215, Dec. 2019.

[18] M. J. Gallant and J. A. Marshall, "Two-dimensional axis mapping using LiDAR," IEEE Trans. on Robotics, vol. 32, no. 1, pp. 150-160, Feb. 2016.

[19] D. Macii, A. Colombo, P. Pivato, and D. Fontanelli, “A Data Fusion Technique for Wireless Ranging Performance Improvement," IEEE Trans. on Instrumentation and Measurement, vol. 62, no. 1, pp. 2737, Jan. 2013.

[20] B. Choi, J. Lee, J. Lee, and K. Park, "A hierarchical algorithm for indoor mobile robot localization using rfid sensor fusion," IEEE Transactions on Industrial Electronics, vol. 58, no. 6, pp. 2226-2235, Jun. 2011.

[21] A. Motroni, P. Nepa, V. Magnago, A. Buffi, B. Tellini, D. Fontanelli, and D. Macii, "SAR-based Indoor Localization of UHF-RFID Tags via Mobile Robot," in International Conference on Indoor Positioning and Indoor Navigation (IPIN). Nantes, France: IEEE, Sep. 2018, pp. 1-8.

[22] A. Buffi, D. Fontanelli, D. Macii, V. Magnago, A. Motroni, P. Nepa, and B. Tellini, "UHF-RFID Localization: The Problem of Antenna Phase Center in Phase-based Methods," in 2019 13th European Conference on Antennas and Propagation (EuCAP), Krakow, Poland, 2019, pp. 1-5.

[23] E. DiGiampaolo and F. Martinelli, "A Passive UHF-RFID System for the Localization of an Indoor Autonomous Vehicle," IEEE Transactions on Industrial Electronics, vol. 59, no. 10, pp. 3961-3970, Oct. 2012.

[24] L. Yang, J. Cao, W. Zhu, and S. Tang, "Accurate and Efficient Object Tracking Based on Passive RFID," IEEE Transactions on Mobile Computing, vol. 14, no. 11, pp. 2188-2200, Nov. 2015.

[25] S. S. Saab and Z. S. Nakad, "A Standalone RFID Indoor Positioning System Using Passive Tags," IEEE Transactions on Industrial Electronics, vol. 58, no. 5, pp. 1961-1970, May 2011.

[26] S. Park and H. Lee, "Self-Recognition of Vehicle Position Using UHF Passive RFID Tags," IEEE Transactions on Industrial Electronics, vol. 60, no. 1, pp. 226-234, Jan. 2013.

[27] X. Song, X. Li, W. Tang, W. Zhang, and B. Li, “A Hybrid Positioning Strategy for Vehicles in a Tunnel Based on RFID and In-Vehicle Sensors," Sensors, vol. 14, no. 12, pp. 23 095-23 118, 2014.

[28] A. Buffi, P. Nepa, and F. Lombardini, "A phase-based technique for localization of UHF-RFID tags moving on a conveyor belt: Performance analysis and test-case measurements,' IEEE Sensors Journal, vol. 15, no. 1, pp. 387-396, Jan. 2015.

[29] A. Buffi, A. Motroni, P. Nepa, B. Tellini, and R. Cioni, "A SAR-based measurement method for passive-tag positioning with a flying UHFRFID reader,' IEEE Transactions on Instrumentation and Measurement, vol. 68, no. 3, pp. 845-853, Mar. 2019.

[30] P. V. Nikitin, R. Martinez, S. Ramamurthy, H. Leland, G. Spiess, and K. V. S. Rao, "Phase based spatial identification of uhf rfid tags," in 2010 IEEE International Conference on RFID (IEEE RFID 2010), Orlando, FL, USA, Apr. 2010, pp. 102-109.

[31] A. Tzitzis, S. Megalou, S. Siachalou, E. Tsardoulias, A. Kehagias, T. Yioultsis, and A. G. Dimitriou, "Localization of RFID Tags by a Moving Robot, via Phase Unwrapping and Non-Linear Optimization," IEEE Journal of Radio Frequency Identification, vol. 3, no. 4, pp. 216226, Dec. 2019.

[32] E. DiGiampaolo and F. Martinelli, "Mobile Robot Localization Using the Phase of Passive UHF RFID Signals," IEEE Transactions on Industrial Electronics, vol. 61, no. 1, pp. 365-376, Jan. 2014.

[33] F. Martinelli, "A Robot Localization System Combining RSSI and Phase Shift in UHF-RFID Signals," IEEE Transactions on Control Systems Technology, vol. 23, no. 5, pp. 1782-1796, Sep. 2015.

[34] A. Motroni, A. Buffi, P. Nepa, and B. Tellini, "A Phase-based Method for Mobile Node Localization through UHF-RFID passive tags," in 2019 IEEE International Conference on RFID Technology \& Application (RFID-TA), Pisa, Italy, Sep. 2019, pp. 470-475.

[35] V. Magnago, L. Palopoli, D. Fontanelli, D. Macii, A. Motroni, P. Nepa, A. Buffi, and B. Tellini, "Robot localisation based on phase measures of backscattered UHF-RFID signals," in 2019 IEEE International Instrumentation and Measurement Technology Conference (I2MTC), Auckland, New Zealand, May 2019, pp. 1-6.

[36] A. Bekkali, H. Sanson, and M. Matsumoto, "RFID Indoor Positioning Based on Probabilistic RFID Map and Kalman Filtering," in Third IEEE International Conference on Wireless and Mobile Computing, Networking and Communications (WiMob 2007), Oct. 2007, pp. 2121.

[37] Chi-Tsong Chen, Linear system theory and design. Oxford University Press, Inc., 1998.

[38] A. Isidori, Nonlinear control systems. Springer Science \& Business Media, 2013.

[39] S. J. Julier and J. K. Uhlmann, "New extension of the kalman filter to nonlinear systems," in Signal processing, sensor fusion, and target recognition VI, vol. 3068, Jul. 1997, pp. 182-193.

[40] A. I. A. Krener, "Linearization by output injection and nonlinear observers," Syst. Control Lett.3, vol. 1, p. 4752, 1983.

[41] P. Bevilacqua, M. Frego, E. Bertolazzi, D. Fontanelli, L. Palopoli, and F. Biral, "Path planning maximising human comfort for assistive robots," in Proc. 2016 IEEE Conference on Control Applications (CCA), Buenos Aires, Argentina, Sep. 2016, pp. 1421-1427. 